\title{
Plasmonic biosensors fabricated by galvanic displacement reactions for monitoring biomolecular interactions in real time
}

\author{
Claudia Pacholski $^{1}$ - Sophia Rosencrantz ${ }^{2}$. Ruben R. Rosencrantz ${ }^{2}$ - Ruth Fabiola Balderas-Valadez ${ }^{1}$
}

Received: 27 September 2019 / Revised: 1 January 2020 / Accepted: 13 January 2020 / Published online: 31 January 2020

(C) The Author(s) 2020

\begin{abstract}
Optical sensors are prepared by reduction of gold ions using freshly etched hydride-terminated porous silicon, and their ability to specifically detect binding between protein $\mathrm{A} /$ rabbit IgG and asialofetuin/Erythrina cristagalli lectin is studied. The fabrication process is simple, fast, and reproducible, and does not require complicated lab equipment. The resulting nanostructured gold layer on silicon shows an optical response in the visible range based on the excitation of localized surface plasmon resonance. Variations in the refractive index of the surrounding medium result in a color change of the sensor which can be observed by the naked eye. By monitoring the spectral position of the localized surface plasmon resonance using reflectance spectroscopy, a bulk sensitivity of $296 \mathrm{~nm} \pm 3 \mathrm{~nm} / \mathrm{RIU}$ is determined. Furthermore, selectivity to target analytes is conferred to the sensor through functionalization of its surface with appropriate capture probes. For this purpose, biomolecules are deposited either by physical adsorption or by covalent coupling. Both strategies are successfully tested, i.e., the optical response of the sensor is dependent on the concentration of respective target analyte in the solution facilitating the determination of equilibrium dissociation constants for protein $\mathrm{A} /$ rabbit IgG as well as asialofetuin/Erythrina cristagalli lectin which are in accordance with reported values in literature. These results demonstrate the potential of the developed optical sensor for cost-efficient biosensor applications.
\end{abstract}

Keywords Optical sensor $\cdot$ Gold nanostructure $\cdot$ Localized surface plasmon resonance $\cdot$ Surface functionalization $\cdot$ Biomolecular interactions $\cdot$ Lectin

\section{Introduction}

The optical phenomenon of surface plasmon resonance (SPR) has been exploited for sensor applications for decades and is based on a collective oscillation of the electron gas in certain materials including gold and silver [1]. Localized surface plasmon resonance (LSPR) in gold nanoparticles, which can be directly excited by

Published in the topical collection Advances in Direct Optical Detection with guest editors Antje J. Baeumner, Günter Gauglitz, and Jiri Homola.

Electronic supplementary material The online version of this article (https://doi.org/10.1007/s00216-020-02414-0) contains supplementary material, which is available to authorized users.

Ruth Fabiola Balderas-Valadez

balderas@uni-potsdam.de; ruth.balderas@uaem.mx

1 Institute of Chemistry, University of Potsdam, Karl-Liebknecht-Straße 24-25, 14476 Potsdam, Germany

2 Fraunhofer Institute for Applied Polymer Research IAP, Biofunctionalized Materials and (Glyco)Biotechnology, Geiselbergstraße 69, 14476 Potsdam, Germany light, was found to be highly sensitive to refractive index changes in close proximity to the gold surface (up to $\sim 10 \mathrm{~nm}$ ). It provokes a selective absorption and scattering of photons and the spectral position as well as the intensity of the optical response depend in this case not only on the refractive index of the surrounding medium but also on the size, shape, and material of the metallic nanoparticles facilitating the realization of tailor-made optical sensors [2]. A large variety of fabrication strategies for LSPR sensors have been developed, which can be divided in the employment of either top-down or bottom-up methods as well as a combination of both. Here, top-down methods rely on the fabrication of masks by rather sophisticated methods, for example, e-beam lithography, which facilitate the formation of highly defined nanostructures upon subsequent deposition of gold using physical vapor deposition or other appropriate techniques for creating thin metallic films [3]. Bottom-up strategies are mainly based on wet-chemically synthesized building blocks which are often arranged on appropriate substrates [4] or in hydrogel using self-assembly [5]. However, plasmonic nanoparticles have also been investigated as optical sensors directly in solution by e.g. exploiting controlled aggregation based on chemical interactions [6]. The synthesis of metallic 
nanoparticles is well-established today and encompasses chemical [7], photochemical [8], and biological routes [9]. The research on LSPR sensors is a very active area and nowadays encompass the optimal arrangement of gold nanostructures on sensor surfaces for providing optimal analyte transport $[10,11]$, the combination plasmonic structures with other materials (e.g., fluorescent quantum dots for surface-enhanced fluorescence) [12], miniaturization [13], and surface-enhanced Raman spectroscopy leading to the detection of single molecules [14]. However, simple and cost- and timeefficient fabrication strategies for highly sensitive LSPR sensors utilizing shifts in the spectral position of the LSPR for signal transduction were most often fabricated by deposition of wetchemically prepared gold nanoparticles on substrate surfaces. The benefits of directly using the substrate for preparing plasmonic nanostructures have barely been investigated. One easy and fast approach to do so is based on the growth of metallic nanostructures on semiconductor surfaces via galvanic displacement. The phenomenon was described e.g. by Alia et al. in 2014 [15]. Galvanic displacement occurs when a "template" (metal or semiconductor) comes into contact with a more noble cation (where nobility is related to the standard redox potential). In this case, it is thermodynamically favorable for the more noble cation to "steal" electrons from the less noble template. The galvanic displacement may occur spontaneously or may be promoted by a third agent. For example, in order to promote the growth of gold nanoparticles or thin layers of gold on silicon oxide surfaces, potassium tetrachloroaurate (III) $\left(\mathrm{KAuCl}_{4}\right)$ is offered in solution which also contains a certain percentage of hydrofluoric acid (HF). The parameters of the reaction, the influence of the template orientation, and the proposed reaction mechanism have been nicely reviewed by Lahiri and Kobayashi in 2016 [16]. Similar surfaces may be fabricated by spontaneous galvanic displacement of gold cations on hydrogenated silicon. In this case, the presence of HF was not required. It was proposed that such spontaneous reaction is related to the distribution of charges in the $[\equiv \mathrm{Si}-\mathrm{H}]$ group [17]. Nevertheless, even if for none of the two cases the mechanism is completely understood, this technique is useful, reproducible, and for a long time used in industry $[18,19]$ for the fabrication of ohmic junctions and Schottky barriers. However, the realization of LSPR sensors by galvanic displacement reactions has barely been reported until now, even though substrates for surfaceenhanced Raman spectroscopy (SERS) [20-22] or surfaceenhanced infrared spectroscopy (SEIRAS) [23, 24] were often prepared in this way.

LSPR sensors are tunable platforms as they detect refractive index changes in close proximity to the sensor surface and are most often used without special labels. Hence, any analyte which has an affinity to the metallic sensor surface will provoke an optical response. To obtain a high selectivity for detecting certain biomolecules and to investigate their binding kinetics, appropriate capture probes for the intended target analyte have to be presented on the sensor surface. For this purpose, different functionalization protocols have been reported ranging from physical adsorption and covalent coupling to complex formation [25]. Between all the possibilities, the functionalization with proteins is, probably, the one that is most often chosen in biosensing experiments due to their extraordinary recognition capacity. For example, glucose oxidase is an enzyme that recognizes and reacts with glucose and commercial sensors are based on this reaction to measure glucose concentrations in blood [26]. One known and useful recognition element for testing the ability of sensors to monitor biomolecular interactions in real time is Protein A $(\operatorname{PrA})$, a protein found in the cell wall of the bacterium Staphylococcus aureus, which plays an important role in the virulence of such bacteria by binding to antibodies [27]. The PrA may be bound to metallic surfaces in order to coffer them a high selectivity for antibodies, especially certain immunoglobulin $\mathrm{G}(\mathrm{IgG})$. It has been shown that highly sensitive LSPR biosensors can be obtained by well-ordered nanostructures functionalized with PrA $[28,29]$. Another application field is the recognition of glycan structures. Glycans are found on every living higher cell and enable communication between a cell and its surroundings [30]. They appear as glycolipids, as proteoglycans, or as glycoproteins. Glycosylation is a complex posttranslational modification due to many different enzymes involved in this process. The structural complexity of oligosaccharides allows information-coding. Their counterparts are specific glycan recognition proteins, so-called lectins [31]. In this context, the asialofetuin/Erythrina cristagalli lectin interaction can be used as model system. Methods like SPR, ITC (isothermal titration calorimetry), MST (microscale thermophoresis), or ELLA (enzyme-linked lectin assay) can be explored for measuring carbohydrate binding events, on the one hand to understand biological processes and on the other hand for glycan analysis. LSPR sensors using glycopolymers, gold nanoparticles, or selfassembled monolayers are already described for lectin binding studies [32-36]. However, for providing LSPR sensors by mass production, both a well thought through functionalization strategy of the sensor surface with the desired capture probes and a time- and cost-efficient fabrication method are required.

In this work, a simple and fast fabrication strategy for LSPR sensors using galvanic displacement reactions in combination with cost-efficient surface functionalization methods is presented, and the potential of the resulting nanostructured gold layers on silicon substrates for monitoring biomolecular interactions is demonstrated.

\section{Materials and methods}

\section{Materials}

Silicon wafers (p-type, $0.001-0.002 \Omega \mathrm{cm},<100>$ ) were obtained from Siegert Wafer GmbH (Germany). Hydrofluoric acid (48\%), 2-propanol, and toluene were purchased from Merck. Ethanol (99.8\%), glutardialdehyde, and hydroxyethyl 
piperazineethanesulfonic acid (HEPES) were supplied by Carl Roth $\mathrm{GmbH}+\mathrm{Co} . \mathrm{KG}$ (Germany). Galvanic displacement reactions were carried out in ethanol (96\%) supplied by VWR International GmbH (Germany) and with $\mathrm{HAuCl}_{4} \cdot 3$ $\mathrm{H}_{2} \mathrm{O}$ (99.99\%) obtained from Alfa Aesar (Thermo Fisher (Kandel) GmbH, Germany). Cysteamine, Protein A, rabbit $\mathrm{IgG}$, PBS buffer, bovine serum albumin, asialofetuin, and acetic acid were supplied by Sigma. Erythrina cristagalli lectin was purchased from Vector Laboratories via BIOZOL Diagnostica Vertrieb GmbH (Eching, Germany). Hydrochloric acid $(\mathrm{HCl})$ was supplied by Th. Geyer.

\section{Fabrication of sensors based on gold nanostructures}

First, a sacrificial layer of porous silicon was prepared by electrochemically etching of p-type silicon wafer pieces (0.001-0.002 $\Omega \mathrm{cm}$, orientation $\langle 100\rangle$, Siegert Wafer). Etching was carried out using an electrolyte containing ethanol and hydrofluoric acid at $48 \%$ in a ratio 1:1 (v:v). A current density of $133 \mathrm{~mA} \mathrm{~cm}{ }^{-1}$ was applied for $67 \mathrm{~s}$ using a Kepco Power Supply. Freshly etched porous silicon samples were immediately immersed in a $2 \mathrm{mM}$ solution of $\mathrm{HAuCl}_{4}$. $3 \mathrm{H}_{2} \mathrm{O}$ dissolved in a 1:2 (w:w) mixture of ethanol and MilliQ water. This gold nanostructures growth reaction was carried out at a controlled temperature of $31^{\circ} \mathrm{C}$. After a reaction time of $7 \mathrm{~min}$, the samples were removed from the gold salt solution, washed extensively with ethanol, and dried in a stream of $\mathrm{N}_{2}$. Subsequently, the samples were incubated in basic solution (15 mM NaOH dissolved in a 1:1 (w:w) mixture of ethanol:water) overnight. The basic solution was removed in the morning, and the samples let dry in air. Details of the optimization process for preparing nanostructured gold layers using galvanic displacement reactions can be found in the Electronic Supplementary Material (ESM) of this article.

\section{Scanning electron microscopy}

Scanning electron micrographs were obtained with a Zeiss Ultra 55 "Gemini" scanning electron microscope (Carl Zeiss, Inc., Oberkochen, Germany), which was operated at an accelerating voltage of $10.0 \mathrm{keV}$. Backscattered electrons were detected for obtaining the presented micrographs.

\section{Optical characterization}

An Ocean Optics, Inc. (USA) charged-coupled device (CCD) spectrometer (model Flame) was utilized for collecting reflectance spectra. For this purpose, a bifurcated optical fiber was equipped with a microscope objective lens and connected to both the spectrometer and a tungsten light source. Through the microscope objective lens a spot with a size of $\sim 1-2 \mathrm{~mm}^{2}$ was illuminated with light. Reflectivity spectra were recorded from $400-1000 \mathrm{~nm}$ with a spectral acquisition time of $5.7 \mathrm{~ms}$ and a total integration time of $\sim 10 \mathrm{~s}$ resulting from averaging five spectral scans. Reflectivity spectra were collected at normal incidence. In order to obtain reflectance spectra, the reflectivity spectrum of the respective sample was divided by a reflectivity reference spectrum which was collected previously from an aluminum mirror.

\section{Determination of the sensor sensitivity}

The bulk sensitivity in $\mathrm{nm} / \mathrm{RIU}$ (refractive index unit) of the nanostructured gold layer (nAuL) was determined by measuring the shift in the wavelength of the LSPR (minimum of the plasmonic feature in the reflectance spectrum, referred from here as " $\lambda_{\mathrm{P}}$ ") in response to the refractive index $(n)$ of the medium surrounding the sensor. For this purpose, the reflectance spectra of the nAuL were recorded in air $(n=1)$ and after being immersed in three different organic solvents with different refractive index, namely, ethanol $(n=1.361)$, 2-propanol $(n=1.375)$, and toluene $(n=1.497)$. The difference between the position of the LSPR in liquid and air $\left(\lambda_{\mathrm{P}, n \neq 1}-\lambda_{\mathrm{P}, n=1}\right)$ was calculated and plotted versus the $n$ of the organic solvents. The slope of the linear relationship corresponds to the sensitivity of the sensor.

\section{Covalent binding of protein $A$ to nanostructured gold}

Protein A (PrA) was covalently bound to the sensor surface inspired by the method of Boujday et al. [37] and represented in Fig. 1a. For this purpose, the nAuL was incubated in a $10 \mathrm{mM}$ aqueous solution of cysteamine for $12 \mathrm{~h}$, then washed with MilliQ water and dried in a stream of $\mathrm{N}_{2}$. The resulting amine-terminated sensor surface was submerged in a $0.1 \mathrm{M}$ ethanolic solution of glutardialdehyde for $12 \mathrm{~h}$, subsequently washed with ethanol, and dried with $\mathrm{N}_{2}$. In order to bind PrA, a solution of $0.1 \mathrm{mg} \mathrm{ml}^{-1}$ of PrA in PBS buffer was flown over the sensor surface. Details can be found in the following "Real-time monitoring of biomolecular interactions" section.

\section{Physical adsorption of Protein A to the nanostructured gold layer}

PrA was adsorbed to the nAuL without any previous functionalization as is shown in Fig. 1b. For this purpose, a solution of $0.1 \mathrm{mg} \mathrm{ml}^{-1}$ of PrA in PBS buffer was flown over the sensor surface. Experimental details of the attachment to the gold surface can be found in the following "Real-time monitoring of biomolecular interactions" section.

\section{Real-time monitoring of biomolecular interactions}

The interaction of biomolecules with the sensor surface was monitored using specular reflectance spectroscopy and by following the shift in $\lambda_{\mathrm{P}}$, which is caused by the adsorption or 


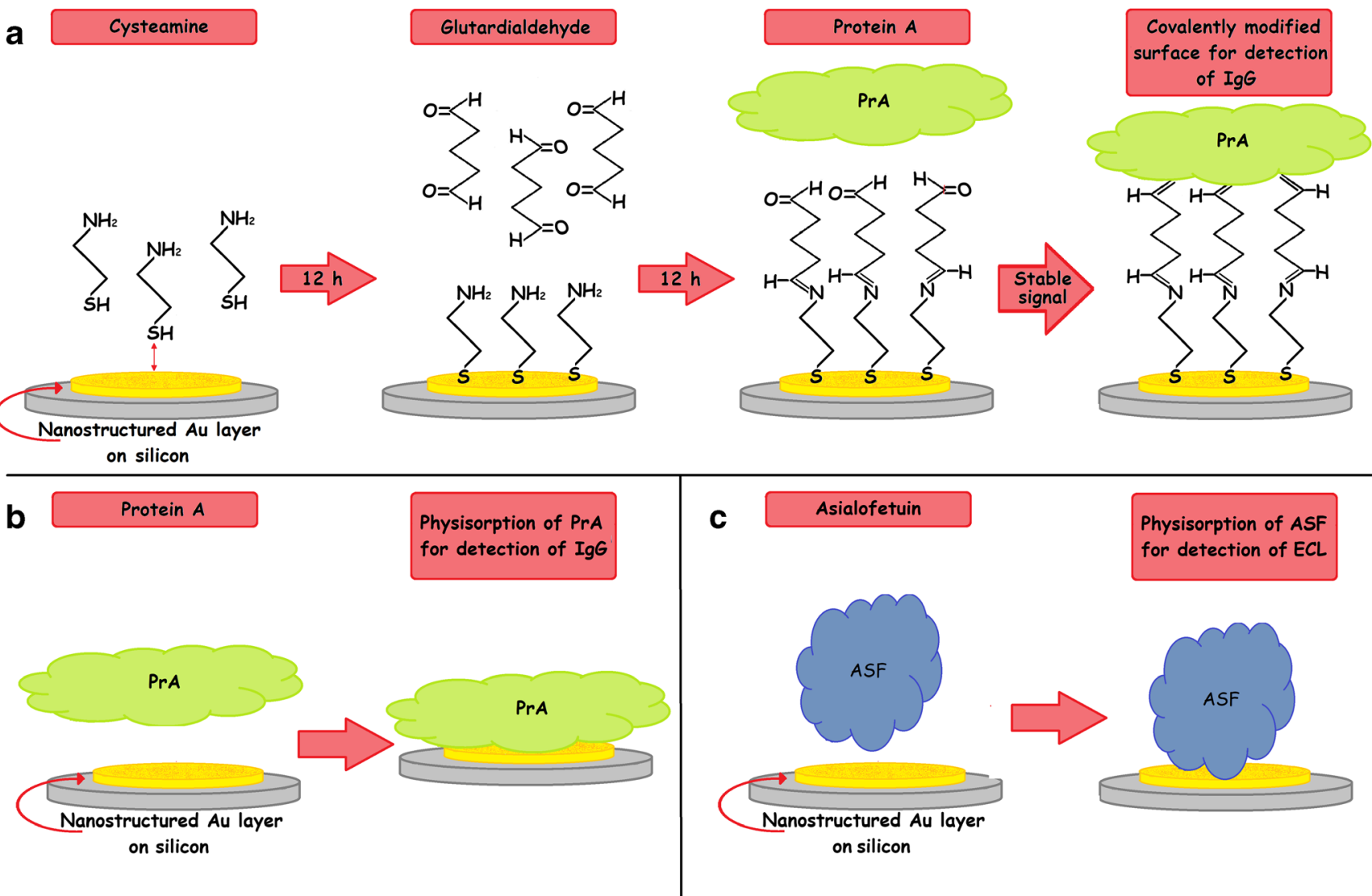

Fig. 1 Scheme of the investigated surface functionalization strategies: a metallic surface covalently modified with PrA, b physisorption of PrA on the $\mathrm{nAuL}$, and $\mathbf{c}$ physisorption of ASF on the $\mathrm{nAuL}$

binding of the biomolecule to the sensor surface. For this purpose, the nAuL was fixed inside a custom-made flow cell (plexiglass). Light was guided to and from sensor surface via a bifurcated optical fiber through the plexiglass cover and reflectance spectra were collected in regular intervals. Different solutions were flown over the sensor surface using a peristaltic pump (Perimax 12, SPETEC) and a flow rate of $0.57 \mathrm{ml} \mathrm{min}^{-1}$.

For functionalizing the sensor surface with $\operatorname{PrA}$ (with and without previous chemical modification), different solutions were successively introduced into the flow cell. First, PBS buffer $(\mathrm{pH}=7.4)$ was flown through the cell for $10 \mathrm{~min}$ in order to establish a base line. Afterwards, $5 \mathrm{ml}$ of a PBS buffer solution containing $0.1 \mathrm{mg} / \mathrm{ml}$ of $\operatorname{PrA}(42 \mathrm{kDa})$ were recirculated through the flow cell. Attachment of the PrA to the $\mathrm{nAuL}$ provoked a shift in $\lambda_{\mathrm{P}}$. The recirculation of the $\operatorname{PrA}$ was continued until the $\lambda_{\mathrm{P}}$ did not change anymore. Pure PBS buffer was introduced into the cell for $10 \mathrm{~min}$ in order to replace the PrA solution. Then, a $0.1 \mathrm{M}$ aqueous solution of acetic acid was passed over the nAuL to remove PrA from the sensor surface which was not strongly attached to the sensor surface. Finally, the sensor was exposed to PBS buffer again and was ready for biomolecular interaction studies.
The interactions of the PrA-modified sensors with immunoglobulins (IgGs) and bovine serum albumin (BSA) were studied directly after their functionalization. For this purpose, a solution of $0.1 \mathrm{mg} / \mathrm{ml}$ of immunoglobulin $\mathrm{G}$ (IgG, $150 \mathrm{kDa})$ from rabbit in PBS was flown through the flow cell, also provoking a shift in the $\lambda_{\mathrm{P}}$ due to the association of rabbit IgG to PrA. After obtaining a constant value of $\lambda_{\mathrm{P}}$ PBS buffer was passed over the sensor surface in order to monitor the dissociation of rabbit IgG from PrA. To recover the sensor surface, a $0.1 \mathrm{M}$ aqueous solution of acetic acid was introduced into the flow cell, followed finally by an exposure to PBS buffer. This cycle was repeated 4 times using different concentrations of IgG. As negative control, a solution of $1 \mathrm{mg} / \mathrm{ml}$ of BSA in PBS buffer was flown over the sensor surface.

\section{Biomolecular interaction of the system ASF/ECL}

In order to demonstrate the capability of the developed optical sensor to also monitor other biomolecular interactions, a second capture probe/target molecule combination, namely, asialofetuin (ASF, $48 \mathrm{kDa}$ ) and Erythrina cristagalli lectin (ECL, $54 \mathrm{kDa}$ ), was investigated (Fig. 1c). For this purpose, 
freshly prepared nAuL sensors without functionalization were fixed inside the custom-made flow cell and reflectance spectra were recorded in real time again. The sensor was first exposed to lectin buffer (10 mM HEPES, $150 \mathrm{mM} \mathrm{NaCl}, 0.1 \mathrm{mM}$ $\mathrm{CaCl2}, \mathrm{pH} 7.5)$ for $10 \mathrm{~min}$. Afterwards, a solution of $0.25 \mathrm{mg} / \mathrm{ml}$ of the glycoprotein ASF in lectin buffer was recirculated through the flow cell until a stable value of $\lambda_{\mathrm{P}}$ was observed. Then, the ASF solution was replaced with a solution of $1 \mathrm{mg} / \mathrm{ml}$ of BSA in lectin buffer. To remove loosely attached proteins from the sensor surface, a $0.1 \mathrm{M}$ aqueous solution of $\mathrm{HCl}$ was passed over the sensor. The interaction of ECL with ASF was then monitored by successively introducing the following solutions into the flow cell: lectin buffer, ECL in different concentrations (dissolved in lectin buffer), lectin buffer, $0.1 \mathrm{M}$ aqueous $\mathrm{HCl}$, and lectin buffer.

\section{Results and discussion}

\section{Fabrication and characterization of plasmonic sensors}

In Fig. 2a, a representative scanning electron micrograph (SEM) of a fabricated $\mathrm{nAuL}$ is displayed. The $\mathrm{nAuL}$ was obtained by immersing freshly etched porous silicon in a solution containing $\mathrm{Au}^{3+}$ ions. Here, hydrides covering the surface of freshly etched porous silicon reduce $\mathrm{Au}^{3+}$ ions leading to the formation of a nanostructured gold layer on top of the porous silicon. The formed $\mathrm{nAuL}$ is composed of polydisperse nanoparticles with different shapes, which are mainly non-spherical. Moreover, longer gold nanorods are randomly dispersed over the whole surface. The growth of similar gold nanostructures on silicon by galvanic displacement reactions was already reported $[16,38$, 39]. The gold nanostructures were exclusively located on the top surface of the porous silicon layer and no gold could be observed in the pores by inspecting cross-sectional SEMs. The appearance of this nAuL did not change after the removal of the porous silicon underneath using a basic solution.

The formation of nanostructured gold layers on freshly etched porous silicon was monitored by collecting reflectance spectra at normal incidence. A reflectance spectrum of a freshly etched porous silicon layer is characterized by an interference pattern resulting from the superposition of light rays reflected at the interfaces of the porous silicon layer. After immersion of the freshly etched porous silicon in a solution containing $\mathrm{Au}^{3+}$ ions, gold nanoparticles start to grow at the porous silicon surface leading to the appearance of a valley in the interference pattern. In Fig. 2b (I), a representative reflectance spectrum of a porous silicon layer with gold nanostructures on top after an immersion time of $7 \mathrm{~min}$ is displayed. The valley located at $\sim 600 \mathrm{~nm}$ and indicated with the red arrow is caused by the excitation of localized surface plasmon resonance in the deposited gold nanoparticles [21]. If the porous silicon underneath the gold nanoparticle layer is removed by dissolution in basic solution, only this valley was observed in the reflectance spectrum (red arrow in Fig. 2b (II)) in accordance with published spectra for gold nanoparticles on silicon substrates [40].

\section{Plasmonic sensor characterization and functionalization}

The position of the characteristic LSPR signal in the reflectance spectrum shifts to smaller or longer wavelengths depending on the refractive index of the surrounding medium. The bulk sensitivity of the plasmonic sensor can be obtained by considering the magnitude of this shift and is quantified in $\mathrm{nm} / \mathrm{RIU}$ (refractive index unit). In Fig. 3a, the difference between the spectral position of the LSPR signal resulting from the nAuL immersed in liquids with different refractive indices and the spectral position of the LSPR signal of the gold nanoparticle layer in air (" $\lambda_{\mathrm{P}, n \neq 1}$ $\left.-\lambda_{\mathrm{P}, n=1}\right)$ is plotted versus the refractive index of the surrounding medium. Seven different plasmonic sensors were investigated for this purpose and a bulk sensitivity of $296 \mathrm{~nm} \pm 3 \mathrm{~nm} / \mathrm{RIU}$ was determined by calculating the slope of the linear relationship. The determined sensitivity is in the upper region for LSPR-based optical sensors [41]. The small standard deviation indicates the high reproducibility of the presented fabrication strategy for plasmonic sensors using galvanic displacement reactions. Also, the shift in the $\lambda_{\mathrm{P}}$ due the change in the $n$ of the surrounding medium can be observed by the naked eye. Photographs of the plasmonic sensors immersed in different liquids are displayed in Fig. 3b. In air $(n=1)$, the structure shows a vivid green color; after being
Fig. 2 Characterization of fabricated plasmonic sensors: a SEM of the nAuL on top of the porous silicon layer, $\mathbf{b}$ reflectance spectra of a nAuL with (I) and without (II) porous silicon underneath
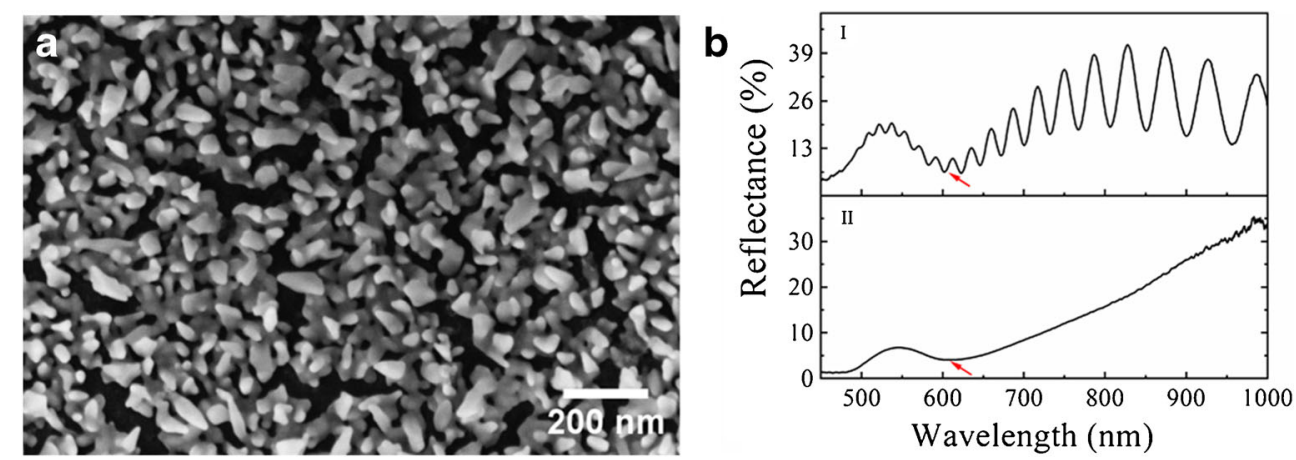
Fig. 3 Properties of plasmonic sensors: a determination of the bulk sensitivity by plotting the spectral position of the LSPR signal versus the refractive index of the surrounding medium, $\mathbf{b}$ photographs of the plasmonic sensor in air and organic solvents with different $n$

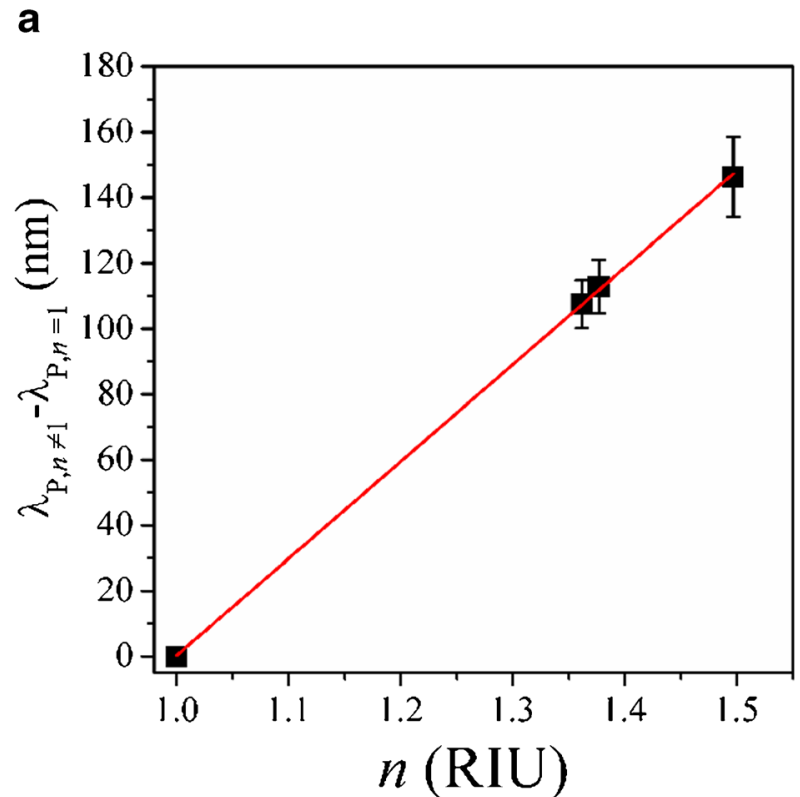

b

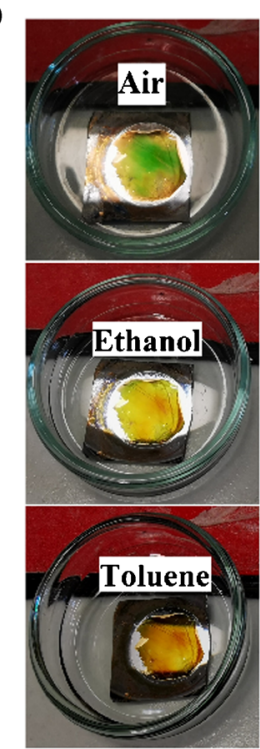

immersed in ethanol ( $n=1.361)$, the color of the surface changed to yellow and in toluene $(n=1.497)$ to a reddish color.

To obtain an amino-terminated sensor surface suitable for covalent binding of biomolecules using glutardialdehyde, the gold nanoparticle layer was immersed in a $10 \mathrm{mM}$ aqueous solution of cysteamine for $12 \mathrm{~h}$. Cysteamine forms a selfassembled monolayer on the $\mathrm{nAuL}$ in which the thiol is bound to the gold and the amine group is presented to the surrounding medium [42]. In Fig. 4a, a representative SEM of a nAuL after cysteamine treatment is shown. In comparison to the nAuL before functionalization (Fig. 2a), the gold nanostructures size significantly decreased and the morphology of the nanostructures appears smoother (for better comparison please check the ESM: Fig. S6). Similar observations have been reported for gold nanoparticles immersed in solutions with high cysteamine concentrations $[43,44]$. The reduction of the gold nanoparticle size in the fabricated plasmonic sensors leads also to changes in their optical properties - as expected. The spectral position of the LSPR signal in the reflectance spectrum of the plasmonic sensor is shifted to lower wavelengths as shown in Fig. 4b. In air and before functionalization, the plasmon resonance is located approximately at $600 \mathrm{~nm}$. After immersion of the sensor in an aqueous solution of cysteamine, the LSPR shifted to a $\lambda_{\mathrm{P}} \approx 700 \mathrm{~nm}$ as is expected due the change in the $n$ of the surrounding medium. After $12 \mathrm{~h}$ incubation in the cysteamine solution, the plasmon resonance can be found to be at a $\lambda_{\mathrm{P}} \approx 540 \mathrm{~nm}$ (still immersed in aqueous solution). The changes in the spectral position of the LSPR can be caused by a reduction in the size of the gold nanostructures. However, after the functionalization and when the structure is dried, the valley in the reflectance spectra, caused by LSPR, cannot be easily observed anymore. This might be explained by a further shift of the LSPR resonance to shorter wavelengths which have not been investigated. Nevertheless, if the refractive index in the surrounding medium changes from air to e.g. buffer solution, the LSPR can be well detected again. Finally, the amineterminated sensor surface was submerged in a $0.1 \mathrm{M}$ ethanolic solution of glutardialdehyde for $12 \mathrm{~h}$ in order to allow for covalently coupling of biomolecules. In this case, free aldehyde groups react with amine groups present in the biomolecules.
Fig. 4 Characterization of the $\mathrm{nAuL}$ after cysteamine functionalization. a SEM of the gold nanostructures after functionalization with cysteamine, and $\mathbf{b}$ reflectance spectra of plasmonic sensor before and after its functionalization with cysteamine. Red arrows highlight the position of the LSPR feature
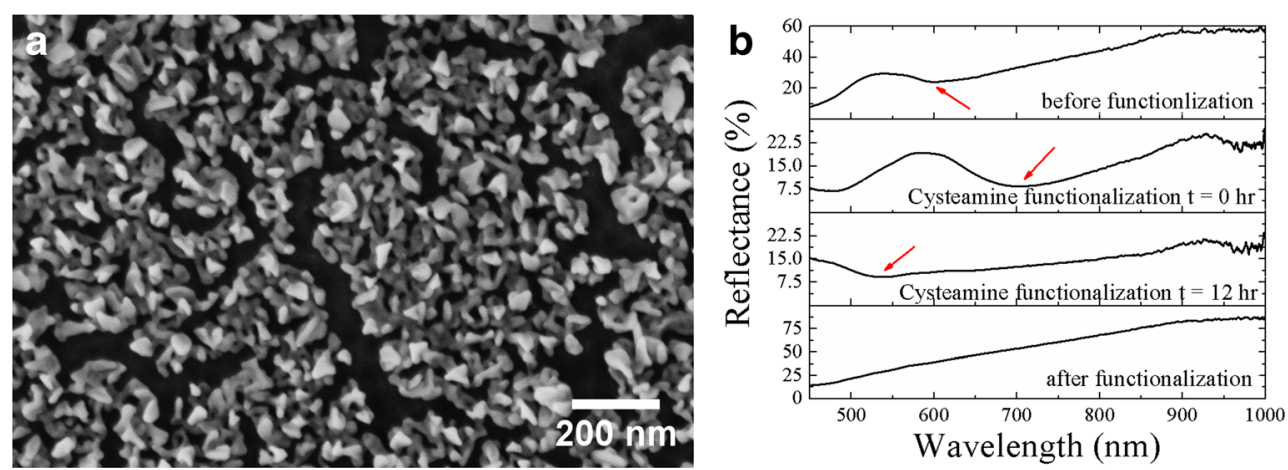


\section{Real-time monitoring of biomolecular interactions}

Two different model systems were investigated, namely, protein A (PrA)/rabbit IgG and asialofetuin (ASF)/ Erythrina cristagalli lectin (ECL), for studying the performance of the fabricated plasmonic biosensor regarding real-time monitoring of biomolecular interactions. PrA was first isolated from the cell wall of the bacteria Staphylococcus aureus and can bind antibodies [27]. Therefore, it was and still is often utilized for the separation, immobilization and detection of immunoglobulins. ECL is a plant lectin with a high selectivity for binding to carbohydrate moieties of glycoproteins and glycolipids [45]. Lectins are responsible for cellular recognition, adhesion, signal transduction, and metastasis. ASF is a standard glycoprotein presenting terminal galactose residues that can be bound by lectins like ECL [46].

\section{Protein A—rabbit IgG interactions}

First, the biosensor surface had to be equipped with PrA. For this purpose, plasmonic sensors with and without cysteamine/ glutardialdehyde functionalization were treated in the same way. Both were fixed in a custom-made flow cell and successively exposed to different solutions, namely, PBS buffer, a solution of $0.1 \mathrm{mg} / \mathrm{ml}$ PrA, PBS buffer, $0.1 \mathrm{M}$ aqueous acetic acid solution, PBS buffer. The deposition of PrA was followed by collecting reflectance spectra and following the spectral position of the LSPR signal (Fig. 5).

The optical response of the plasmonic sensor to covalent coupling of PrA to the sensor surface functionalized with cysteamine/glutardialdehyde is shown in Fig. 5a. In the first $10 \mathrm{~min}$, PBS was flown over the sensor surface in order to establish a stable baseline. Afterwards, PrA was introduced into the flow cell leading to a shift in the spectral position of the LSPR signal due to an increase of the refractive index at the sensor surface. The magnitude of the shift was determined in triplicate - from three different plasmonic sensors - to be $2.8 \mathrm{~nm} \pm 0.2 \mathrm{~nm}$ demonstrating the high reproducibility of the fabrication process as well as of the functionalization strategy. The PrA solution was recirculated through the flow cell until no significant shift in the LSPR signal position could be detected anymore. Then, the sensor was exposed to an aqueous $0.1 \mathrm{M}$ acetic acid solution in order to remove unbound PrA. The spectral position of the LSPR signal shifted to shorter wavelengths indicating the removal of adsorbed PrA from the sensor surface. Finally, PBS buffer was introduced into the flow cell leading to a small optical response of the sensor which is based on the difference between the refractive index of PBS buffer and of aqueous $0.1 \mathrm{M}$ acetic acid solution. Overall, the covalent binding of PrA to the sensor surface previously functionalized with cysteamine and glutardialdehyde resulted in a change of $\sim 2 \mathrm{~nm}$ in the spectral position of the LSPR signal (sensor immersed in buffer before

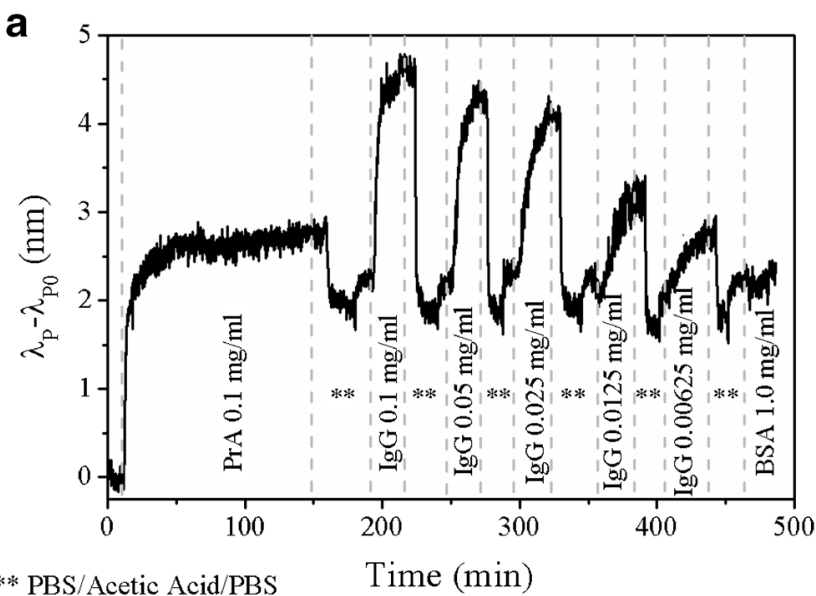

** PBS/Acetic Acid/PBS Time (min)
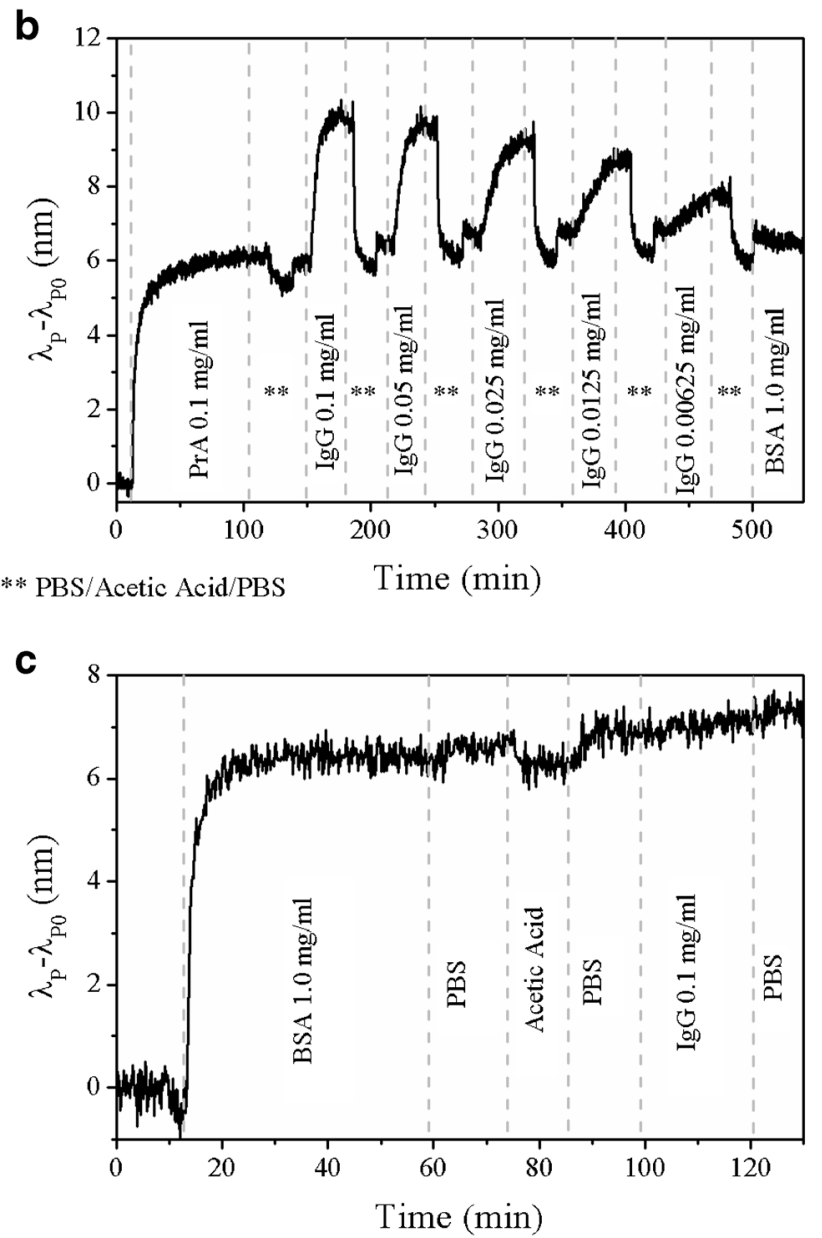

Fig. 5 Optical response of plasmonic sensors to deposition of PrA and successive exposure to rabbit IgG solutions: a results for plasmonic sensor with covalently bound PrA, b results for plasmonic sensor with physically adsorbed PrA, $\mathbf{c}$ observed spectral shifts of plasmonic sensors with physically adsorbed BSA

and after deposition of PrA). Similar experiments were carried out using plasmonic sensors without functionalization, i.e., PrA was adsorbed directly to the gold surface. The optical response of the sensor to PrA adsorption is shown in Fig. 5b. A shift of $7.0 \mathrm{~nm} \pm 0.7 \mathrm{~nm}$ in the spectral position of the 
LSPR signal was determined from triplicate measurements. We speculate that the difference in the magnitude of the shifts due to the deposition of PrA by physical adsorption or covalent attachment on the plasmonic sensors can be explained by the distance of the biomolecule to the sensor surface and the changes in the gold nanostructure caused by reaction with cysteamine. In the first case, cysteamine/glutardialdehyde increase the distance of PrA to the gold surface. As the electromagnetic field of LSPR has a very short, exponentially decreasing decay length, this additional distance between gold surface and PrA should be one explanation for the smaller shift [47]. Furthermore, the treatment of the gold nanostructure with cysteamine changed its morphology leading to a shift of the LSPR signal to shorter wavelengths and to a somehow smoother gold surface. Both could result in a lower sensitivity of the plasmonic sensor due to the wavelength dispersion of the refractive index and the available surface area.

Directly after deposition of PrA, the plasmonic sensors were exposed to solutions with different concentrations of rabbit IgG in PBS buffer in order to evaluate the capability of the sensors to monitor biomolecular interactions in real time. PrA is known to bind strongly to rabbit IgG [48]. The optical response of the plasmonic sensors functionalized with PrA either by covalent binding or physical adsorption showed an optical response to the presence of rabbit IgG (Fig. 5a, b, respectively). Here, the magnitude of the spectral shifts of the LSPR signal depended on the chosen functionalization strategy and on the concentration of the antibody in PBS buffer: at higher concentrations of rabbit $\mathrm{IgG}$, the shift was larger in comparison to lower concentrations of rabbit IgG. The difference in the observed spectral shifts for plasmonic sensors with covalently bound or physically adsorbed PrA can be explained in a similar way as before described in detail for the deposition of PrA. To test for non-specific binding, a solution of BSA in PBS buffer was also flown over the sensor surface which did not lead to changes in the spectral position of the LSPR signal demonstrating the selectivity of the plasmonic sensors. Also, it is important to highlight that even if the spectral shifts due to the biomolecular interactions at the sensor surface are higher for the plasmonic sensor with physically adsorbed PrA, the stability of its base line, i.e., the ability to return to the same spectral position after the association, dissociation, and removal of rabbit $\mathrm{IgG}$ from the sensor surface, is better for the plasmonic sensor with covalently bound PrA. An explanation for this behavior might be based on the prevention of detachment of PrA during drastic changes in $\mathrm{pH}$ by the covalent bonds between PrA and sensor surface. In the case of physically adsorbed PrA, desorption might occur during the experiment, opening up space for non-specific binding between the sensor surface and rabbit $\operatorname{IgG}[25]$. It is noteworthy, that in the case of covalent binding using glutardialdehyde it is often highly recommended to block non-reacted aldehyde groups with ethanolamine or glycine in order to prevent non-specific binding of analyte molecules to the sensor surface [49].
A negative control was carried out by physically adsorbing BSA to the plasmonic sensor surface and by successively flow a solution with a high concentration of rabbit $\operatorname{IgG}$ in PBS buffer over its surface. In Fig. 5c, the optical response to both the deposition of BSA and the exposure to rabbit $\operatorname{IgG}$ are shown. As expected, the adsorption of BSA to the sensor surface resulted in a significant spectral shift of the LSPR signal due to the change in the refractive index caused by BSA. Exposure of the plasmonic sensor to rabbit $\mathrm{IgG}$ did not result in an optical response indicating that the spectral shift of the LSPR signal caused by rabbit IgG in Fig. 5a, b is due to its binding to PrA instead of changes in the refractive index in the surrounding medium or non-specific interactions between the sensor surface and rabbit IgG.

The sensorgrams also provided information on the association and dissociation of rabbit $\operatorname{IgG}$ to/from protein $\mathrm{A}$ and facilitated the kinetic analysis of the biomolecular interaction in order to extract thermodynamic equilibrium binding constants. For this purpose, the spectral shift of the LSPR signal was determined by:

$\Delta \lambda=\lambda_{I g G}-\lambda_{0}$

where $\lambda_{0}$ is the spectral position of the LSPR signal after deposition of PrA in PBS buffer and $\lambda_{\mathrm{IgG}}$ is the spectral position of the LSPR signal at equilibrium of rabbit IgG binding to the sensor surface at different concentrations. In Fig. $6, \Delta \lambda$ is plotted versus the concentration of rabbit IgG for plasmonic sensors functionalized by physical adsorption or covalent

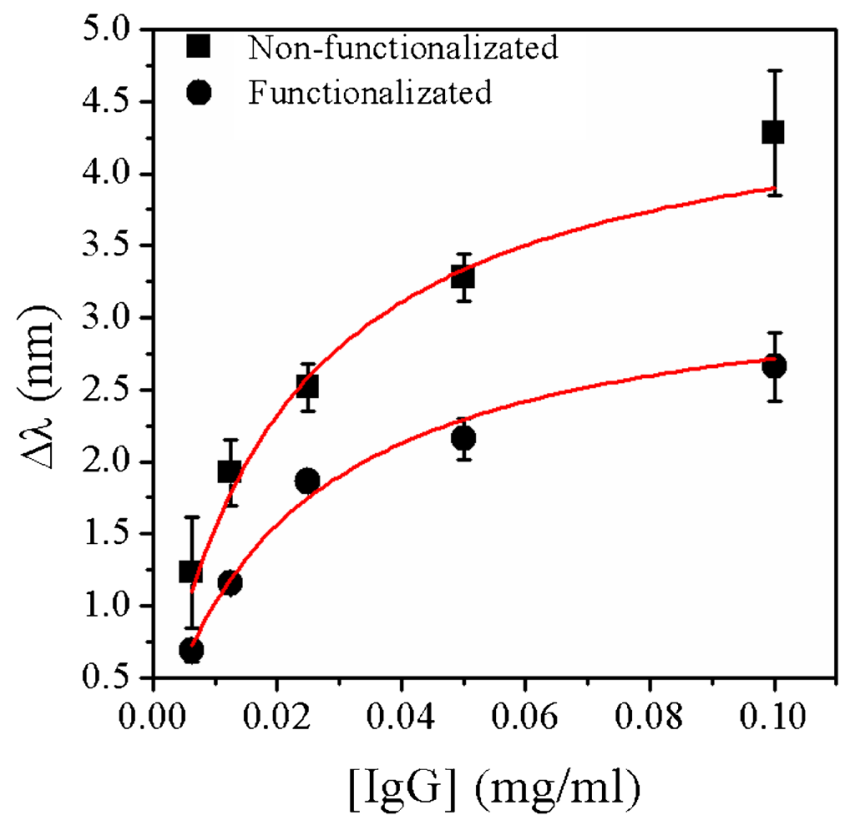

Fig. 6 Dependency of the spectral position of the LSPR signal on the offered concentration of rabbit IgG in PBS buffer. Values are shown for three trials using three separately fabricated plasmonic sensors which were functionalized with PrA by physical adsorption or covalent coupling. Solid lines correspond to fitting these values to a Langmuir isotherm for determining equilibrium dissociation constants ( $K_{\mathrm{D}}$ values) 
coupling of PrA to the sensor surface. For extracting the equilibrium dissociation constant $K_{D}$, the average values of three trials are fitted to a Langmuir isotherm $[50,51]$ resulting in values of $K_{D}=1.5 \times 10^{-7} \mathrm{M} \pm 2.7 \times 10^{-8} \mathrm{M}$ and $K_{D}=1.4 \times$ $10^{-7} \mathrm{M} \pm 2.8 \times 10^{-8} \mathrm{M}$ for the sensors functionalized by covalent coupling or physical adsorption of PrA, respectively. These $K_{\mathrm{D}}$ values are in accordance with reported ones for similar studies [52].

\section{ASF-ECL interactions}

To demonstrate the generality of the capabilities of the presented plasmonic sensor to monitor biomolecular interactions in real time, another model system, namely, ASF/ ECL, was also investigated. Biosensor experiments were performed again by collecting reflectance spectra of the sensor during exposure to various solutions in a flow cell. To avoid size reduction of the nanostructures in the $\mathrm{nAuL}$ and a decrease in the response of the sensor [53], no previous chemical functionalization was performed for this system and the attachment of the glycoprotein to the metallic surface was achieved by physical adsorption. The spectral shifts of the LSPR signal indicate the interactions of biomolecules with the sensor surface (Fig. 7a). The spectral shift due to the physical adsorption of ASF to the metallic sensor surface was $11.9 \mathrm{~nm} \pm 0.9 \mathrm{~nm}$. As was expected, BSA did not provoke an optical response of the plasmonic sensor because it does interact with the glycoprotein. The association of ECL to ASF led to spectral shifts of the LSPR signal whose magnitude was related to the concentration of the lectin in the solution. The dependency of the spectral shifts on the concentration of ECL in solution is plotted in Fig. $7 \mathrm{~b}$ for three trials using three separately fabricated plasmonic sensors. A $K_{\mathrm{D}}$ value of $4.9 \times 10^{-7} \mathrm{M} \pm 1.7 \times 10^{-8} \mathrm{M}$ was determined for ASF/ECL by fitting the data to a Langmuir isotherm, consistent with published values on ASF [54] and on lactose glycopolymers [55].

\section{Detection limits of the sensors}

The smallest amount of analyte that can be accurately detected and quantified by a sensing device is known as the detection limit (DL). The DL should be expressed in units of concentration (M, $\mathrm{g} \mathrm{L}^{-1}$, etc.) and represents one of the most important performance parameters of a sensor. To evaluate such parameter for the $\mathrm{nAuL}$, the methodology recommended by the IUPAC and explained for Chiavaioli et al. [56] was chosen. This methodology requires a calibration curve of the response of the sensor vs. the concentration of the target analyte at a concentration of 1 to 5 times higher than the suspected DL, an accurate fitting function of such calibration curve, the standard deviation $(\sigma)$ of the blank measurements (measurement without the analyte under investigation), and the mean value of the blank measurement $\left(Y_{\text {blank }}\right)$. Then, the concentration of the detection limit is equal to the inverse of the fitting function evaluated in $\left(Y_{\text {blank }}+3 \sigma\right)$. The nanostructured gold layers were evaluated for the detection of IgG using a modified surface of PrA attached either covalently or physically adsorbed to the metallic surface and ECL using a surface modified with ASF. The DL for the three systems were calculated using as calibration curve the data of Figs. 6 and $7 \mathrm{~b}$ and as fitting function the Langmuir isotherm. The $Y_{\text {blank }}$ has a normalized value of 0 . The magnitudes of the $\sigma \mathrm{s}$ are $0.1 \mathrm{~nm}$ and $0.2 \mathrm{~nm}$, when nanostructured gold layers with and without cysteamine functionalization were used, respectively. The detection limits were calculated to be $0.4 \mu \mathrm{g} / \mathrm{ml}$ for rabbit $\mathrm{IgG}$ detection using covalently coupled protein A on the sensor surface, $0.3 \mu \mathrm{g} / \mathrm{ml}$ for sensors modified with PrA by adsorption, and $0.2 \mu \mathrm{g} / \mathrm{ml}$ for ECL.

Hybrid sensors containing surfaces showing LSPR, which are coupled to optical fibers and report DL in the order of magnitude of $\mathrm{nM}$ or $\mathrm{fM}$, have been reported in recent years. However, their optical setup differs considerably from the one used in the presented nAuL sensor. Table 1 lists newer LSPR sensors that can be compared to the nAuL sensor. First of all, it is important to emphasize that such a comparison can be
Fig. 7 Investigation of ASF-ECL interactions using the fabricated plasmonic sensor: a optical response of the sensor to exposure to different solutions. $\mathbf{b}$ Dependency of the spectral shift of the plasmonic sensor on the concentration of ECL in solution. The solid line represents a fit to a Langmuir isotherm for determining the equilibrium dissociation constant $K_{\mathrm{D}}$
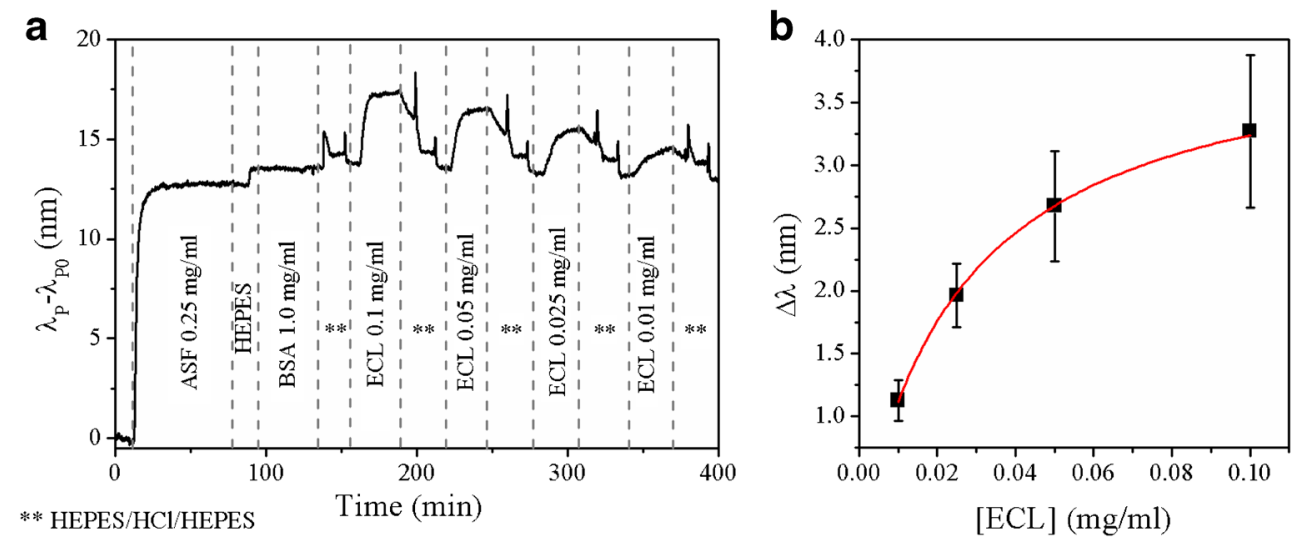
Table 1 LSPR sensors

\begin{tabular}{|c|c|c|c|c|c|}
\hline Structure & $\begin{array}{l}\text { Analysis } \\
\text { methodology }\end{array}$ & Target & Sensitivity & DL & Reference \\
\hline Gold nanoislands & $\begin{array}{l}\text { Absorbance and } \\
\text { TIRE }\end{array}$ & Mycotoxins as AFT B1 & $\begin{array}{l}77.28 \text { and } \\
207 \mathrm{~nm} / \text { RIU* }^{*}\end{array}$ & $0.01 \mathrm{ng} / \mathrm{ml} * *$ & {$[57,58]$} \\
\hline Gold nanoislands & $\begin{array}{l}\text { TIRE/LSPR } \\
\text { (absorption) }\end{array}$ & Mycotoxins as AFT B1 & $5300 \mathrm{rad} / \mathrm{RIU}$ & $0.002 \mathrm{ng} / \mathrm{ml}$ & [59] \\
\hline $\begin{array}{l}\text { Gold arrays fabricated by } \\
\text { interferometric } \\
\text { lithography. }\end{array}$ & Absorbance & $\begin{array}{l}\text { Histidine-tagged green fluorescent } \\
\text { protein (His-GFP) and bacteriochloro- } \\
\text { phyll a (BChl a) }\end{array}$ & $145 \mathrm{~nm} / \mathrm{RIU}$ & - & {$[60]$} \\
\hline $\begin{array}{l}\text { Square array of holes coupled } \\
\text { with an optical cavity. }\end{array}$ & Reflectance & $\begin{array}{l}\text { Iron oxide nanoparticles }(80 / 100 \mathrm{~nm}) \\
\text { and Avidin }\end{array}$ & $0.5 \mathrm{~nm} / \mathrm{nm}^{* * *}$ & $\begin{array}{l}10-3 \mathrm{pM} \text { and } \\
0.5 \mathrm{nM}\end{array}$ & {$[61]$} \\
\hline $\begin{array}{l}\text { Square array of holes } \\
\text { functionalized with } \\
\text { oxygen-deficient cerium } \\
\text { oxide nanoparticles }\end{array}$ & Reflectance & Dopamine in blood & - & $1 \mathrm{nM}$ & {$[62]$} \\
\hline
\end{tabular}

*Calculated by absorbance

**obtained by TIRE

***Analysis made using the shift of the LSPR with respect to the controlled deposition of thin layers of $\mathrm{Al}_{2} \mathrm{O}_{3}$

difficult, since the evaluation of the different parameters as sensitivity or the DL can be calculated by different methods. The unit of sensitivity of a plasmonic system is typically given in $\mathrm{nm} / \mathrm{RIU}$, but depending on the reference, it can also be given as $\mathrm{rad} / \mathrm{RIU}$ [59] or nm/nm *** [60]. Another parameter that the DL can influence is the chosen transduction method. For example, Al Rubaye et al. presented in 2017 [57] gold nanoislands fabricated by annealing gold films that exhibit a maxima sensitivity of $77 \mathrm{~nm} / \mathrm{RIU}$ using absorbance and $207 \mathrm{~nm} / \mathrm{RIU}$ using spectroscopic ellipsometry. In 2019 [59], the same group reported the exploitation of the same kind of sensor surfaces to achieve a higher sensitivity and a lower DL for the detection of mycotoxin by investigating their performance using a combination of total internal reflection ellipsometry (TIRE), LSPR transducer, and planar waveguides operating as polarization interferometer.

Since comparing the performance of different sensors is nowadays mainly achieved by evaluating determined DLs, it is crucial to be aware of the different parameters affecting these values. For example, even today, there are discrepancies about the units and the methodology for the calculation of DLs for a wide range of sensing platforms. Some authors use the equation proposed by White and Fan in 2008 [63] that describes the DL as the resolution of the experiment divided by the sensitivity. Other authors claim that such an approximation is not accurate [64] and that the calculation of DLs should be based on calibration curves. Furthermore, such calibration curves should be preferentially measured using analyte concentrations roughly 1 to 5 time higher than the suspected DL. These requirements resulted from the fact that in literature, commonly lower DL were reported which were calculated from experiments carried out with analyte concentrations much higher than the reported magnitude. Also, another important factor, that has a great repercussion on the magnitude of the DL, is the data treatment. Processes like smoothing reduce the noise of the system. Hence, the standard deviation of the measurements is reduced. Moreover, some authors have demonstrated that the DL of a sensor may be enhanced not by the modification of the transduction material but by the modification or change of the data treatment [65]. Finally, the flexibility and capacity of the external instrumentation (e.g., spectrometers) as well as the parameters used in the detection experiment (acquisition time, integration time, light source, etc.) may have an influence on the determined DL. For all these reasons, DLs alone cannot be easily used for comparing the performance of optical sensors.

\section{Conclusions}

A simple and inexpensive optical biosensor capable of monitoring biomolecular interactions was fabricated by the spontaneous galvanic displacement reaction of $\mathrm{Au}^{3+}$ cations on freshly etched porous silicon covered with hydrogen groups. The reduction of the metal ions led to the formation of a continuous nanostructured gold layer on the porous film. In the reflectance spectrum of the gold/porous silicon hybrid structure, a broad valley at $\sim 604 \mathrm{~nm}$ in air in the interference pattern was observed, which was resulting from the excitation of localized surface plasmon resonance in the gold nanostructures. The isolation of the nanostructured gold layer was achieved by dissolution of the porous silicon matrix using basic solution. The remaining nanostructured gold layer showed a high sensitivity to refractive index changes in the surrounding medium. Changes in the localized surface plasmon resonance were exploited for studying biomolecular interactions in real time. For the latter purpose, two different functionalization strategies were investigated for comparing 
their influence on the sensitivity and stability of the optical sensor response using PrA/rabbit IgG as model system. On the one hand, PrA was directly adsorbed to the metallic surface, and on the other hand, it was covalently attached to the sensor surface using cysteamine/glutardialdehyde. Whereas the shift of the localized surface plasmon resonance is larger for the adsorption of PrA directly to the sensor surface, the stability of the optical response of the sensor is improved in the case of covalently bound PrA. However, both functionalization strategies proved to be sufficient in order to monitor association and dissociation of rabbit IgG to/from PrA and determine accurate equilibrium dissociation constants. Moreover, another type of biomolecular interactions, namely, between ASF/ ECL, was also successfully followed in real time using the developed optical sensor. The DL is relatively high in comparison to other reported sensor. However, neither the conditions for recording the reflectance spectra nor the data treatment was optimized for calculating the DLs of the developed sensor. Hence, the DL shown in here are the possible highest values which can be expected for this sensor. For example, an enhancement of the DL may be achieved by using a more sophisticated data treatment - but this is not the objective of this work. In a nutshell, the presented fabrication strategy for plasmonic biosensors is simple and inexpensive and can provide sensitive optical sensors for investigating biomolecular interactions.

Acknowledgments Open Access funding provided by Projekt DEAL. The authors would like to thank the research group of Prof. Dr. J. P. Spatz and especially Mr. I. Grigoridis at the Max Planck Institute for Medical Research for taking the presented scanning electron micrographs.

Funding information C.P. received financial support via the Heisenberg program from the German Research Foundation (DFG, project number 426213922). R.F.B.V received funding from CONACyT (postdoctoral fellowship, CVU 264841, call number 291258). The contribution of S.R. and R.R.R. was supported as Fraunhofer High Performance Center for Functional lntegration in Materials.

\section{Compliance with ethical standards}

Conflict of interest The authors declare that they have no conflict of interest.

Open Access This article is licensed under a Creative Commons Attribution 4.0 International License, which permits use, sharing, adaptation, distribution and reproduction in any medium or format, as long as you give appropriate credit to the original author(s) and the source, provide a link to the Creative Commons licence, and indicate if changes were made. The images or other third party material in this article are included in the article's Creative Commons licence, unless indicated otherwise in a credit line to the material. If material is not included in the article's Creative Commons licence and your intended use is not permitted by statutory regulation or exceeds the permitted use, you will need to obtain permission directly from the copyright holder. To view a copy of this licence, visit http://creativecommons.org/licenses/by/4.0/.

\section{References}

1. Xu Y, Bai P, Zhou X, Akimov Y, Png CE, Ang L, et al. Optical refractive index sensors with plasmonic and photonic structures: promising and inconvenient truth. Adv Opt Mater. 2019;7(9): 1801433.

2. Unser S, Bruzas I, He J, Sagle L. Localized surface plasmon resonance biosensing: current challenges and approaches. Sensors. 2015;15(7):15684-716.

3. Sperling JR, Macias G, Neale SL, Clark AW. Multilayered nanoplasmonic arrays for self-referenced biosensing. ACS Appl Mater Interfaces. 2018;10(40):34774-80.

4. Feng B, Zhu R, Xu S, Chen Y, Di J. A sensitive LSPR sensor based on glutathione-functionalized gold nanoparticles on a substrate for the detection of $\mathrm{Pb} 2+$ ions. RSC Adv. 2018;8(8):4049-56.

5. Liu D, Fang L, Zhou F, Li H, Zhang T, Li C, et al. Ultrasensitive and stable au dimer-based colorimetric sensors using the dynamically tunable gap-dependent plasmonic coupling optical properties. Adv Funct Mater. 2018;28(18):1707392.

6. Zhu J, Zhao B, Qi Y, Li J-J, Li X, Zhao J-W. Colorimetric determination of $\mathrm{Hg}$ (II) by combining the etching and aggregation effect of cysteine-modified Au-Ag core-shell nanorods. Sensors Actuators B Chem. 2018;255:2927-35.

7. Daruich De Souza C, Ribeiro Nogueira B, MECM R. Review of the methodologies used in the synthesis gold nanoparticles by chemical reduction. J Alloys Compd. 2019;798:714-40.

8. McGilvray KL, Fasciani C, Bueno-Alejo CJ, Schwartz-Narbonne R, Scaiano JC. Photochemical strategies for the seed-mediated growth of gold and gold-silver nanoparticles. Langmuir. 2012;28(46):16148-55.

9. Singh P, Kim YJ, Zhang D, Yang DC. Biological synthesis of nanoparticles from plants and microorganisms. Trends Biotechnol. 2016;34(7):588-99.

10. Lynn NS, Homola J. (Bio)sensing using nanoparticle arrays: on the effect of analyte transport on sensitivity. Anal Chem. 2016;88(24): 12145-51.

11. Špačková B, Lynn NS, Slabý J, Šípová H, Homola J. A route to superior performance of a nanoplasmonic biosensor: consideration of both photonic and mass transport aspects. ACS Photonics. 2018;5(3):1019-25.

12. Song HY, Wong TI, Sadovoy A, Wu L, Bai P, Deng J, et al. Imprinted gold 2D nanoarray for highly sensitive and convenient PSA detection via plasmon excited quantum dots. Lab Chip. 2015;15(1):253-63.

13. Niu L, Zhang N, Liu H, Zhou X, Knoll W. Integrating plasmonic diagnostics and microfluidics. Biomicrofluidics. 2015;9(5):52611.

14. Aizpurua J, Arnolds H, Baumberg J, Bruzas I, Chikkaraddy R, Chisanga $\mathrm{M}$, et al. Ultrasensitive and towards single molecule SERS: general discussion. Faraday Discuss. 2017;205:291-330.

15. Alia SM, Yan YS, Pivovar BS. Galvanic displacement as a route to highly active and durable extended surface electrocatalysts. Catal Sci Technol. 2014;4(10):3589-600.

16. Lahiri A, Kobayashi S-I. Electroless deposition of gold on silicon and its potential applications: review. Surf Eng. 2016;32(5):32137.

17. Tertykh VA, Katok KV, Yanishpolskii VV. The reduction of gold nanoparticles in the surface layer of modified silica. Russ J Phys Chem A. 2008;82(9):1438-41.

18. Andricacos PC, Uzoh C, Dukovic JO, Horkans J, Deligianni H. Damascene copper electroplating for chip interconnections. IBM J Res Dev. 1998;42(5):567-74.

19. Ting $\mathrm{CH}$, Paunovic M. Selective electroless metal deposition for integrated circuit fabrication. J Electrochem Soc. 1989;136(2):45662. 
20. Lin H, Mock J, Smith D, Gao T, Sailor MJ. Surface-enhanced Raman scattering from silver-plated porous silicon. J Phys Chem B. 2004;108(31):11654-9.

21. Virga A, Rivolo P, Descrovi E, Chiolerio A, Digregorio G, Frascella $\mathrm{F}$, et al. SERS active Ag nanoparticles in mesoporous silicon: detection of organic molecules and peptide-antibody assays. J Raman Spectrosc. 2012;43(6):730-6.

22. Bandarenka HV, Girel KV, Zavatski SA, Panarin A, Terekhov SN. Progress in the development of SERS-active substrates based on metal-coated porous silicon. Materials (Basel). 2018;11(5):1-20.

23. Miyake H, Ye S, Osawa M. Electroless deposition of gold thin films on silicon for surface-enhanced infrared spectroelectrochemistry. Electrochem Commun. 2002;4(12):973-7.

24. Huang B-B, Wang J-Y, Huo S-J, Cai W-B. Facile fabrication of silver nanoparticles on silicon for surface-enhanced infrared and Raman analysis. Surf Interface Anal. 2008;40(2):81-4.

25. Oliverio M, Perotto S, Messina GC, Lovato L, De Angelis F. Chemical functionalization of plasmonic surface biosensors: a tutorial review on issues, strategies, and costs. ACS Appl Mater Interfaces. 2017;9(35):29394-411.

26. Yoo EH, Lee SY. Glucose biosensors: an overview of use in clinical practice. Sensors. 2010;10(5):4558-76

27. Surolia A, Pain D, Islam KM. Protein A: nature's universal antiantibody. Trends Biochem Sci. 1982;7(2):74-6.

28. Polley N, Basak S, Hass R, Pacholski C. Biosensors and bioelectronics fiber optic plasmonic sensors : providing sensitive biosensor platforms with minimal lab equipment. Biosens Bioelectron. 2019;132:368-74.

29. Cetin AE, Etezadi D, Galarreta BC, Busson MP, Eksioglu Y, Altug H. Plasmonic nanohole arrays on a robust hybrid substrate for highly sensitive label-free biosensing. ACS Photonics. 2015;2(8):116774.

30. Gabius H-J, Siebert H-C, André S, Jiménez-Barbero J, Rüdiger H. Chemical biology of the sugar code. ChemBioChem. 2004;5(6): 740-64.

31. Gabius H-J. Cell surface glycans: the why and how of their functionality as biochemical signals in lectin-mediated information transfer. Crit Rev Immunol. 2006;26(1):43-80.

32. Rosencrantz RR, Nguyen VH, Park H, Schulte C, Böker A, Schnakenberg U, et al. Lectin binding studies on a glycopolymer brush flow-through biosensor by localized surface plasmon resonance. Anal Bioanal Chem. 2016;408(20):5633-40.

33. Lazar J, Rosencrantz RR, Elling L, Schnakenberg U. Simultaneous electrochemical impedance spectroscopy and localized surface plasmon resonance in a microfluidic chip: new insights into the spatial origin of the signal. Anal Chem. 2016;88(19):9590-6.

34. Liu X, Zhang Q, Tu Y, Zhao W, Gai H. Single gold nanoparticle localized surface plasmon resonance spectral imaging for quantifying binding constant of carbohydrate-protein interaction. Anal Chem. 2013;85(24):11851-7.

35. Bellapadrona G, Tesler AB, Grünstein D, Hossain LH, Kikkeri R, Seeberger PH, et al. Optimization of localized surface plasmon resonance transducers for studying carbohydrate-protein interactions. Anal Chem. 2012;84(1):232-40.

36. Yonzon CR, Jeoung E, Zou S, Schatz GC, Mrksich M, Van Duyne RP. A comparative analysis of localized and propagating surface plasmon resonance sensors: the binding of concanavalin a to a monosaccharide functionalized self-assembled monolayer. J Am Chem Soc. 2004;126(39):12669-76.

37. Boujday S, Bantegnie A, Briand E, Marnet PG, Salmain M, Pradier $\mathrm{CM}$. In-depth investigation of protein adsorption on gold surfaces: correlating the structure and density to the efficiency of the sensing layer. J Phys Chem B. 2008;112(21):6708-15.

38. Harraz FA, Tsuboi T, Sasano J, Sakka T, Ogata YH. Metal deposition onto a porous silicon layer by immersion plating from aqueous and nonaqueous solutions. J Electrochem Soc. 2002;149(9):C456.
39. Balderas-Valadez RF, Schürmann R, Pacholski C. One spot-two sensors: porous silicon interferometers in combination with gold nanostructures showing localized surface plasmon resonance. Front Chem. 2019;7:593.

40. Humbert C, Pluchery O, Lacaze E, Tadjeddine A, Busson B. Optical spectroscopy of functionalized gold nanoparticles assemblies as a function of the surface coverage. Gold Bull. 2013;46(4): 299-309.

41. Couture M, Zhao SS, Masson J-F. Modern surface plasmon resonance for bioanalytics and biophysics. Phys Chem Chem Phys. 2013;15(27):11190.

42. Wirde M, Gelius U, Nyholm L. Self-assembled monolayers of cystamine and cysteamine on gold studied by XPS and voltammetry. Langmuir. 1999;15(19):6370-8.

43. Wang H, Goodrich GP, Tam F, Oubre C, Nordlander P, Halas NJ. Controlled texturing modifies the surface topography and plasmonic properties of Au nanoshells. J Phys Chem B. 2005;109(22): 11083-7.

44. Shu T, Su L, Wang J, Lu X, Liang F, Li C, et al. Value of the debris of reduction sculpture: thiol etching of Au nanoclusters for preparing water-soluble and aggregation-induced emission-active $\mathrm{Au}(\mathrm{I})$ complexes as phosphorescent copper ion sensor. Anal Chem. 2016;88(11):6071-7.

45. Mandal DK, Brewer CF. Cross-linking activity of the 14-kilodalton / 3-galactoside-specific vertebrate lectin with asialofetuin : comparison with several galactose-specific plant lectins. Biochemistry. 1992;31:8465-72.

46. Tang JSJ, Rosencrantz S, Tepper L, Chea S, Klöpzig S, KrügerGenge A, et al. Functional glyco-nanogels for multivalent interaction with lectins. Molecules. 2019;24(10):1865.

47. Willets KA, Van Duyne RP. Localized surface plasmon resonance spectroscopy and sensing. Annu Rev Phys Chem. 2007;58(1):26797.

48. Goding JW. Use of staphylococcal protein A as an immunological reagent. J Immunol Methods. 1978;20(C):241-53.

49. Frederix F, Bonroy K, Reekmans G, Laureyn W, Campitelli A, Abramov MA, et al. Reduced nonspecific adsorption on covalently immobilized protein surfaces using poly(ethylene oxide) containing blocking agents. J Biochem Biophys Methods. 2004;58(1):67-74.

50. Schuck P. Use of surface plasmon resonance to probe the equilibrium and dynamic aspects of interactions between biological macromolecules. Annu Rev Biophys Biomol Struct. 2002;26(1):54166.

51. Kuriyan J, Konforti B, Wemmer D. The molecules of life: physical and chemical principles. Garland Science. 2009:1008.

52. Pacholski C, Yu C, Miskelly GM, Godin D, Sailor MJ. Reflective interferometric Fourier transform spectroscopy: a selfcompensating label-free immunosensor using double-layers of porous. 2006;128(13):4250-4252.

53. Chen H, Kou X, Yang Z, Ni W, Wang J. Shape- and size-dependent refractive index sensitivity of gold nanoparticles. Langmuir. 2008 May;24(10):5233-7.

54. Scheibe C, Bujotzek A, Dernedde J, Weber M, Seitz O. DNAprogrammed spatial screening of carbohydrate-lectin interactions. Chem Sci. 2011;2(4):770-5.

55. Rosencrantz S, Tang JSJ, Schulte-Osseili C, Böker A, Rosencrantz RR. Glycopolymers by RAFT polymerization as functional surfaces for Galectin-3. Macromol Chem Phys. 2019;220(20): 1900293.

56. Chiavaioli F, Gouveia CAJ, Jorge PAS, Baldini F. Towards a uniform metrological assessment of grating-based optical fiber sensors: from refractometers to biosensors. Biosensors. 2017;7(2):23.

57. Al-Rubaye AG, Nabok A, Tsargorodska A. LSPR biosensor based on nanostructured gold films: detection of mycotoxins. Procedia Technol . 2017;27:131-132. 
58. Al-Rubaye A, Nabok A, Abu-Ali H, Szekacs A, Takacs E. LSPR/ TIRE bio-sensing platform for detection of low molecular weight toxins. Proc IEEE Sensors. 2017:1-3.

59. Nabok A, Al-Rubaye AG, Al-Jawdah AM, Tsargorodska A, Marty JL, Catanante G, et al. [INVITED] Novel optical biosensing technologies for detection of mycotoxins. Opt Laser Technol. 2019;109: 212-21.

60. Tsargorodska A, El Zubir O, Darroch B, Cartron ML, Basova T, Hunter $\mathrm{CN}$, et al. Fast, simple, combinatorial routes to the fabrication of reusable, plasmonically active gold nanostructures by interferometric lithography of self-assembled monolayers. ACS Nano. 2014;8(8):7858-69.

61. Vázquez-Guardado A, Smith A, Wilson W, Ortega J, Perez JM, Chanda D. Hybrid cavity-coupled plasmonic biosensors for low concentration, label-free and selective biomolecular detection. Opt Express. 2016;24(22):25785.

62. Vázquez-Guardado A, Barkam S, Peppler M, Biswas A, Dennis W, Das S, et al. Enzyme-free plasmonic biosensor for direct detection of neurotransmitter dopamine from whole blood. Nano Lett. 2019;19(1):449-54.

63. Zhang GX. Porous silicon: morphology and formation mechanisms. In: Vayenas CG, White RE, Gamboa-Adelco ME, editors. Modern aspects of electrochemistry. Boston, MA: Springer US; 2006. p. 65-133.

64. Loock H-P, Wentzell PD. Detection limits of chemical sensors: applications and misapplications. Sensors Actuators B Chem. 2012;173:157-63.

65. Mariani S, Strambini LM, Barillaro G. Femtomole detection of proteins using a label-free nanostructured porous silicon interferometer for perspective ultrasensitive biosensing. Anal Chem. 2016;88(17):8502-9.

Publisher's note Springer Nature remains neutral with regard to jurisdictional claims in published maps and institutional affiliations. 